\title{
INITIAL-VALUED FIRST-ORDER FUZZY DIFFERENTIAL EQUATION IN BI-LEVEL INVENTORY MODEL WITH FUZZY DEMAND
}

\author{
B. DAS ${ }^{1}$, N. K. MAHAPATRA ${ }^{2}$ and M. MAITI ${ }^{3}$ \\ ${ }^{1}$ Department of Mathematics, Jhargram Raj College \\ Jhargram, W.B, India \\ ${ }^{2}$ Department of Mathematics, Panskura Banamali College \\ Panskura, W.B, India \\ ${ }^{3}$ Department of Applied Mathematics with Oceanology and Computer \\ Programming \\ Vidyasagar University, W.B - 721102 India \\ E-mail: barundas2004@yahoo.co.in
}

Received November 17, 2006; revised April 23, 2007; published online December 1, 2008

\begin{abstract}
In the present paper, two methods for the solution of an initial valued first ordered fuzzy differential equation are presented and applied in a fuzzy EOQ model. The constructed model is a bi-level inventory problem involving wholesalerretailers-customers. The wholesaler buys and sells the item instantaneously to several retailers. In the next level, the retailers sell the units to customers with a time dependent imprecise demand, which introduce the fuzzy nature in the differential equation. The selling price of the item is a step-wise time dependent decreasing function. The fuzzy objectives are transformed into crisp one following fuzzy extension principle and centroid formula. The model is illustrated through Interactive Fuzzy Decision Making (IFDM) and Multi Objective Genetic Algorithm (MOGA) and the results from two methods are compared.
\end{abstract}

Key words: Fuzzy differential equation, Bi-level inventory, Newsboy problem, Interactive fuzzy decision making method, Multi-Objective Genetic Algorithm.

\section{Introduction}

In 1965, the first publication in fuzzy set theory by Zadeh [16] showed the intention to accommodate uncertainty in the non-stochastic sense. After that Bellman and Zadeh [1] defined a fuzzy decision making problem as the confluence of fuzzy objectives and constraints operated by max-min operators. 
Recently, fuzzy set theory has been applied to several fields like project network, reliability, production planning, inventory control, etc.

The topic on fuzzy differential equations has been rapidly growing in the recent years. The concept of fuzzy differential was introduced by Chang and Dye [3].The initial value problem for fuzzy differential equation has been studied by Seikkala [15]. In multi-objective decision making (MODM) problems, the problem is summarized as follows:

1. The DM has imprecise information;

2. The DM expresses his preferences subjectively,

3. The statistical inference from historical data and their stability is doubtful.

Many authors suggested different methods to deal with these problems. Sakawa and Yano [14] treated this problems by considering the membership functions of those preferences and uses $\alpha$-level cut to defuzzy the Fuzzy MODM problem and solve it a by using a suitable technique Dey and Maiti [6] has accept this technique.

Since the development of classical economic order quantity (EOQ) model by Harris [11], a lot of research works on inventory control system has been reported in the literature by several authors (cf. Donaldson [3] Chang and Dye [7]) to make the models more realistic. A real-life inventory control model can not be formulated without uncertainty in the parameters and/or variables. Again, this uncertainty may be defined in stochastic and non-stochastic (fuzzy) sense and attempts are being made to formulate and analyze such models (cf. Lee et. al. [4]), Chiang et. al. [12]).

Gallego and Moon [10] defined the newsboy problem as the tool to decide the stock quantity of an item when there is a single purchasing opportunity before the start of the selling period, and the demand for the item is random. The classical newsboy model assumes that if the order quantity is larger than the realized demand, a single discount is used to sell the excess inventory or that excess inventory is disposed off. On the other hand, if the order quantity is less than demand, then profit is lost. The objective is to find the optimum tradeoff between the risk of overstocking (incurring disposal cost) and the risk of under stocking (losing profit). The newsboy problem is a classical inventory problem that is very significant in terms of both theoretical and practical considerations. Items that can be classified under single-period inventory systems include Christmas trees, new-year greeting cards, and of course daily newspapers. The newsboy problem is often used to aid decision-making in fashion, sporting industry, and apparel industry.

Now-days, the computing methods like Genetic Algorithms (GAs) or Simulated Annealing are used as optimization techniques for the near optimum solution of decision making problems. The genetic algorithms are executed iteratively on a set of coded solutions (called populations) with three operators -selection or reproduction, crossover and mutation. Recently, GAs have been applied in different areas like neural network, numerical optimization, pattern recognition, inventory control (cf. Mondal et. al. [13]). 
In each town, normally there exist several small markets connected with a wholesale market, a wholesaler receives an item, say fish, a particular variety of vegetable, or fruit, etc from outside suppliers early in the morning and almost immediately sells the quantities to the retailers of the small markets. After that, these retailers sell the whole amount of the item for the whole day in their respective markets against their imprecise demand in the localities. This situation is observed in the business of fishes, fruits, vegetables, etc. At wholesaler's levels, the problem is of news-boy type, and at retailers level, the problem is an EOQ model in fuzzy environments.

In this paper, we look at a specific problem of first order fuzzy differential equation with one initial value. Our objective is to circulate an economic way of representing such fuzzy dynamical system to the nonfuzzy system. We want to apply this method to a bi-level inventory model consisting of single wholesaler and multi-retailers and considered in fuzzy environment. The wholesaler replenishes instantaneously and disposes those to the retailers immediately, adopting push-sale, if required. Retailers purchase the items from the wholesalers and sell to the customers in a day-market until the whole quantity is sold. The demand of the goods here are considered as a fuzzy function. So in inventory level a fuzzy differential equation arise. This equation is solved in analytical method. After that a set of objective functions is formulated. It is optimised using the Fast and Elitist Multi-Objective Genetic Algorithm (MOGA) and the Interactive Fuzzy Decision Making (IFDM) method. The model is illustrated with some numerical data and results from two methods are compared. Details of presentation are as follows. First, the model is formulated and solved in fuzzy differential or integral form. Then MOGA is developed and we discuss the method of IFDM. Numerical examples are presented to illustrate the model. Discussion of the model and results have been presented. Finally conclusions and suggestions for future research are given.

\section{Basic Concepts in Fuzzy Sets}

Definition 1 [Fuzzy function]. Let $X$ and $Y$ be the universes and $\widetilde{P}(Y)$ the set of all fuzzy sets in $Y$ (power set), $\widetilde{f}: X \rightarrow \widetilde{P}(Y)$ is a mapping. Then $\widetilde{f}$ is a fuzzy function iff

$$
\mu_{\widetilde{f}(x)}(y)=\mu_{\widetilde{R}}(x, y), \quad \forall(x, y) \in X \times Y
$$

where $\mu_{\widetilde{R}}(x, y)$ is the membership function of the fuzzy relation.

Example 1. Let $X$ be the set of all workers of a plant, $\widetilde{f}$ the daily customer who buy a product and $y$ be the demand of the product item. A fuzzy function could then be constructed as $\widetilde{f}(x)=y$ (cf. Zimmermann [17]).

Definition $2[\alpha$-cut]. The $\alpha$-level set or $\alpha$-cut of a fuzzy set $\widetilde{A}$ of $\mathrm{X}$ is a nonfuzzy set denoted by $A_{\alpha}$ and defined as a subset of $\mathrm{X}$ such that $\mu_{\widetilde{A}}(x) \geq \alpha$, i.e. $A_{\alpha}=\left\{x: \mu_{\widetilde{A}}(x) \geq \alpha\right.$ and $\left.x \in X\right\}$. 
Definition 3 [Fuzzy extension principle]. Let $X$ be a Cartesian product of universes $X=X_{1}, X_{2}, \ldots, X_{r}$ and $\widetilde{A}_{1}, \widetilde{A}_{2}, \ldots, \widetilde{A}_{r}$ be fuzzy sets in $X_{1}, X_{2}, \ldots, X_{r}$ respectively. Assume that $f$ is a mapping from $X$ to a universe $Y, y=$ $f\left(x_{1}, \ldots, x_{r}\right)$. Then the extension principle allows us to define a fuzzy set $\widetilde{B}$ in $Y$ by

$$
\left.\widetilde{B}=\left\{\left(y, \mu_{\widetilde{B}}(y)\right): y=f\left(x_{1}, \ldots, x_{r}\right),\left(x_{1}, \ldots, x_{r}\right) \in X\right)\right\},
$$

where

$$
\mu_{\widetilde{B}}(y)=\left\{\begin{array}{l}
\sup _{x \in f^{-1}(y)} \mu_{\widetilde{A}}(x), \quad \text { if } \quad f^{-1}(y) \neq \emptyset \\
0 \quad \text { otherwise. }
\end{array}\right.
$$

Example 2. Let $\widetilde{A}=\{(-1,0.5),(0,0.8),(1,1),(4,0.4)\}$ and $f(x)=x^{2}$. Then by applying the extension principle we get that

$$
\widetilde{B}=f(\widetilde{A})=\{(0, .8),(1,1),(4, .4)\} .
$$

Definition 4 [Fuzzy differential equation]. Let us examine a system whose state at the given moment $t$ may only be described by the fuzzy set $X(t) \in E^{n}$. If someone else was fortunate enough to describe the relation between system state and its rate of change as a function $f: R \times E^{n} \rightarrow E^{n}$, then in the sense of FIFO we come to the notion of the first order fuzzy differential equation

$$
\frac{d \widetilde{X}(t)}{d t}=f(t, \widetilde{X}(t)) \quad \widetilde{X}(0)=\widetilde{k}
$$

Solution Procedure 1. According to Friedman, Ming and Kandel [9], in order to solve initial value problem (2.1), we find out a general solution of the differential equation without initial condition, i.e. we get $\widetilde{X}(t)=g(\widetilde{c}, t)$, where $\widetilde{c}$ is a constant coming from the first-order differential equation. Then $\widetilde{c}$ is derived from the initial condition $\widetilde{X}(0)=\widetilde{k}$.

Solution Procedure 2. According to Buckley [2], first find out the solution $\bar{X}(t)$ of the crisp differential equations $\frac{d \bar{X}(t)}{d t}=f(t, \bar{X}(t))$, with initial condition $\widetilde{X}(0)=\widetilde{k}$.

Then following the knowledge of $\alpha$-cut, the solution of the fuzzy differential equation will be $\left[X_{L}(t), \bar{X}(t), X_{R}(t)\right]$, where

$$
\begin{aligned}
& X_{L}(t)=\min \left\{\tilde{X}(\alpha, t): \mu_{\tilde{X}}(\bar{X})=1, \mu_{\tilde{X}}\left(\bar{X}-\Delta_{L} \bar{X}\right)=0\right\}, \\
& X_{R}(t)=\max \left\{\tilde{X}(\alpha, t): \mu_{\tilde{X}}(\bar{X})=1, \mu_{\tilde{X}}\left(\bar{X}+\Delta_{R} \bar{X}\right)=0\right\} .
\end{aligned}
$$

\subsection{Interactive Fuzzy Decision Making (IFDM) technique}

Considering the imprecise nature of the decision maker's (DM's) judgements, it is natural to assume that the DM may have fuzzy or imprecise goals for each of the objective functions $\max \left(f_{1}(x), f_{2}(x), f_{3}(x), \ldots, f_{m}(x)\right)$. Let a goal 
assigned by the DM to an objective is stated as "somewhat larger than $A$ ". This type of a statement can be quantified by eliciting a corresponding membership function.

To derive the membership function $\mu_{f_{r}(x)}$ for each of the objective functions $f_{r}(x),(r=1,2,3, \ldots, m)$ we first calculate individual minimum $f_{r}^{\min }(x)$ and maximum $f_{r}^{\max }(x)$ values under the given constraints. With the help of these individual minimum and maximum values, the DM can select his membership functions from different types of membership functions (i.e., linear, quadratic, exponential etc.). The membership function for each of the objective functions $f_{r}(x),(r=1,2,3, \ldots, m)$ may be written as

$$
\mu_{f_{r}}(x)= \begin{cases}0, & \text { for } L_{r}>f_{r}(x) \\ d_{r}\left(f_{r}(x)\right), & \text { for } L_{r} \leq f_{r}(x) \leq U_{r} \\ 1, & \text { for } f_{r}(x)>U_{r}\end{cases}
$$

where $L_{r}$ and $U_{r}$ are chosen such that $f_{r}^{\min }(x) \leq L_{r} \leq U_{r} \leq f_{r}^{\max }(x), d_{r}\left(f_{r}(x)\right)$ is a strictly monotone increasing continuous function of $f_{r}(x)$ which may be linear or non-linear.

Type 1. Linear membership function. For each objective function, the corresponding linear membership functions are as follows:

$$
\mu_{f_{r}}(x)= \begin{cases}0, & \text { for } L_{r}>f_{r}(x) \\ 1-\frac{U_{r}-f_{r}(x)}{U_{r}-L_{r}}, & \text { for } L_{r} \leq f_{r}(x) \leq U_{r} \\ 1, & \text { for } f_{r}(x)>U_{r}\end{cases}
$$

It is given in Fig. 1(a).

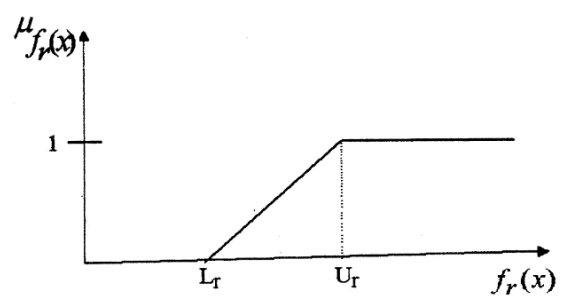

a)

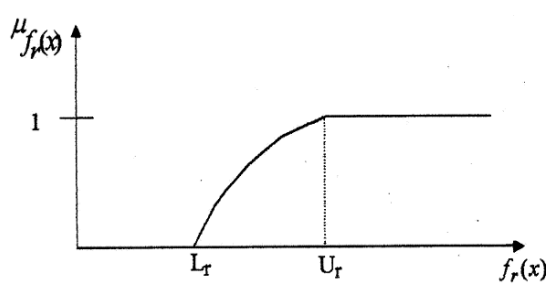

b)

Figure 1. Different types of membership functions: a) linear, b) quadratic.

Type 2. Quadratic membership function. For each objective function, the corresponding quadratic membership functions are as follows:

$$
\mu_{f_{r}}(x)= \begin{cases}0, & \text { for } L_{r}>f_{r}(x), \\ 1-\left(\frac{U_{r}-f_{r}(x)}{U_{r}-L_{r}}\right)^{2}, & \text { for } L_{r} \leq f_{r}(x) \leq U_{r} \\ 1, & \text { for } f_{r}(x)>U_{r} .\end{cases}
$$


It is given in Fig. 1(b).

Type 3. Exponential membership function. For each objective function, the corresponding exponential membership functions are as follows:

$$
\mu_{f_{r}}(x)= \begin{cases}0, & \text { for } L_{r}>f_{r}(x), \\ \alpha_{r}\left[1-e^{\left.-\beta_{r} \frac{U_{r}-f_{r}(x)}{U_{r}-L_{r}}\right],}\right. & \text { for } L_{r} \leq f_{r}(x) \leq U_{r} \\ 1, & \text { for } f_{r}(x)>U_{r} .\end{cases}
$$

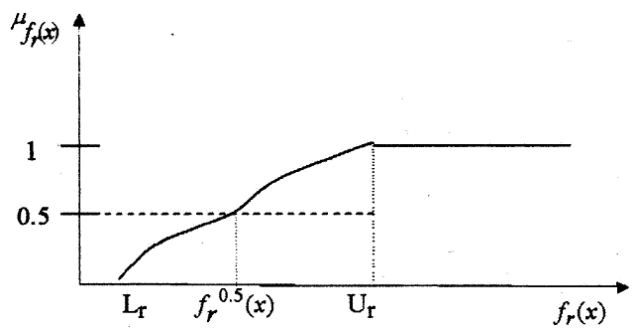

Figure 2. Exponential membership function.

The constants $\alpha_{r}>1$ and $\beta_{r}>0$ can be determined by asking the DM to specify the three points $L_{r}, f_{r}^{0.5}(x)$ and $U_{r}$ such that $f_{r}^{\min }(x) \leq L_{r} \leq f_{r}^{0.5}(x) \leq$ $U_{r} \leq f_{r}^{\max }(x)$ where $f_{r}^{0.5}(x)$ represents the value of $f_{r}(x)$ such that the degree of membership function $\mu_{f_{r}}(x)$ is 0.5 . After determining the different linear/non-linear membership functions for each of the objective functions, following Zimmermann [17] the given problem can be formulated as:

$$
\begin{array}{ll} 
& \max \lambda \\
\text { subject to } & \lambda \leq \mu_{f_{r}}(x), \quad x \in S, \quad 0<\lambda \leq 1, r=1,2,3, \ldots, m .
\end{array}
$$

Now the DM will select the membership functions for the corresponding objective functions. With the help of three different types of membership functions given by (3.6)-(3.8), above problem can be restated as

$$
\begin{aligned}
& \max \lambda \\
\text { subject to } & \lambda \leq \mu_{f_{r}}(x), \quad(\text { if } r \text {-th objective } \in \text { Type } 1) \\
\text { or } & \lambda \leq \mu_{f_{r}}(x), \quad(\text { if } r \text {-th objective } \in \text { Type } 2) \\
\text { or } & \lambda \leq \mu_{f_{r}}(x), \quad(\text { if } r \text {-th objective } \in \text { Type } 3) \\
& x \in S, \quad 0<\lambda \leq 1 .
\end{aligned}
$$

\subsection{Preference to DM's Objective function}

Now the DM will selects his most important objective function from objective functions $f_{r}(x),(r=1,2,3, \ldots, m)$. If it is the $p$-th objective function, then 
problem (3.4), (3.5) is reduced to find $\lambda=\lambda^{*}$ ( $\lambda^{*}$ is an optimal solution of (3.4) and $(3.5))$.

$$
\begin{aligned}
& \max _{\text {s.t. } D} f_{p}(x), \\
& D=\left\{f_{r}(x) \leq \mu_{r}, x \in S, r=1,2,3, \ldots, m\right\},
\end{aligned}
$$

where

$$
\mu_{r}=\left\{\begin{array}{l}
L_{r}+\left(U_{r}-L_{r}\right)\left(1-\lambda^{*}\right), \text { for Type } 1 \text { membership function, } \\
L_{r}+\left(U_{r}-L_{r}\right) \sqrt{\left(1-\lambda^{*}\right)}, \text { for Type } 2 \text { membership function } \\
L_{r}+\frac{1}{\beta_{r}}\left(U_{r}-L_{r}\right) \log \left(1-\frac{\lambda^{*}}{\alpha_{r}}\right), \text { for Type } 3 \text { membership function. }
\end{array}\right.
$$

This new problem may be solved using a single objective optimization technique. Now the $\alpha$ level-curves $\mu_{\widetilde{f}(x)}(y)=\alpha$ for all $\alpha \in[0,1]$ and $\alpha$ and $x$ as parameter have exactly two continuous curves $y=f_{\alpha}^{+}(x)$ and $y=f_{\alpha}^{-}(x)$ for $\alpha \neq 1$ and only one for $\alpha=1$. $f_{\alpha}^{+}(x)$ and $f_{\alpha}^{-}(x)$ are defined such that, $f_{\alpha^{\prime}}^{+}(x) \geq f_{\alpha}^{+}(x) \geq f(x) \geq f_{\alpha}^{-}(x) \geq f_{\alpha^{\prime}}^{-\prime}(x)$ for all $\alpha^{\prime} \geq \alpha$. The integral of any continuous $\alpha$-level curve of $\tilde{f}$ over [a,b] always exist.

Definition 5 [Integration of fuzzy function]. Let $\tilde{f}(x)$ be a fuzzy function from $[a, b] \subseteq R$ to $R$, i.e., $\widetilde{f}(x): \bar{f}(x)$ is the curve for which $\mu_{\tilde{f}(x)}(f(x))=1$ and for $f_{\alpha}^{-}$and $f_{\alpha}^{+}$respectively $\mu_{\widetilde{f}(x)}\left(f_{\alpha}^{-}\right)=0, \mu_{\widetilde{f}(x)}\left(f_{\alpha}^{+}\right)=0$. Then integral of $\widetilde{f}(x)$ over $[a, b]$ is defined to be the fuzzy set

$$
\widetilde{I}(a, b)=\left\{\left(\int_{a}^{b} f_{\alpha}^{-}(x) d x+\int_{a}^{b} f_{\alpha}^{+}(x) d x\right), \alpha\right\} .
$$

This definition is consistent with the extension principle according to which

$$
\mu_{\int_{a}^{b} f}(y)=\sup _{g \in Y} \inf _{x \in[a, b]} \mu_{f(x)}(g(x)), \quad y \in R, \quad g \in Y, \quad x \in[a, b],
$$

where $Y=\{g:[a, b] \rightarrow R, g$ is integrable $\}$ (see, Dubois and Prade [8]).

The determination of the integral $\widetilde{I}(a, b)$ becomes easier if the fuzzy function is assumed to be the LR type. In our paper the fuzzy demand is assumed exponentially over the time interval $\left[0, t_{i}\right]$ which follow the definition of LR fuzzy function, so it has the form $\widetilde{f}(x)=(f(x), l(x), r(x))$. Dubois and Prade [8] have shown that under these conditions

$$
\widetilde{I}(a, b)=\left\{\int_{a}^{b} f(x) d x, \int_{a}^{b} l(x) d x, \int_{a}^{b} r(x) d x\right\} .
$$

Then, it is sufficient to integrate the mean value and the spread functions of $\widetilde{f}(x)$ over $[\mathrm{a}, \mathrm{b}]$ and the result will again be an LR fuzzy number.

\section{Application: Fuzzy Inventory Model}

Here, a single item wholesaler-retailers problem is considered for a single period in fuzzy environment under the following assumptions and notations. 


\subsection{Assumptions}

(i) The replenishment is instantaneous to the wholesaler.

(ii) Demands at retailers' level are time dependent which are assumed to be fuzzy in nature (fuzzy LR-type).

(iii) At retailers' level, the item is sold completely within the time period and at wholesaler's level, units are sold immediately after replenishment using push-sale, if required.

(iv) The item deteriorates, at a constant deterioration rate $\theta$.

(v) Shortages are not allowed to retailers but considered for wholesaler.

(vi) There are $n$ retailers in the system.

Notations. We also will use the following notations: $t_{i}$ denotes the time length of the $i$-th retailer, $\widetilde{Q} / \widetilde{Q}_{i}$ is the order quantity of the wholesaler $/ i$-th retailer, $\widetilde{D_{i}}(t)$ is the fuzzy demand at any time $t$ to the $i$-th retailer, where $\widetilde{D_{i}}(t)=\left(\bar{D}_{i}(t), D_{L i}(t), D_{R i}(t)\right)$, with mean demand $\bar{D}_{i}(t), P$ denotes a unit cost of the item to the wholesaler, $S / S_{a}$ is a normal/push-selling price per unit of the item of the wholesaler, $s_{i}(t)=s_{0 i}\left(1-b_{i}(t) / 100\right)$ is the unit selling price of the retailer at time $t$ with initial price $s_{0 i}$ and positive decreasing rate $b_{i}$, $h_{i} / h_{w}$ is the unit inventory holding cost of the $i$-th retailer/wholesaler, $g_{i} / g_{w}$ is the unit deteriorating cost of the $i$-th retailer/wholesaler, $c_{2 w}$ denotes the unit shortage cost of the wholesaler, $\delta$ is a fraction amount of stock and push-sale / shortage for wholesaler, $\eta_{i}$ is a fraction amount of stock to be received by the $i$-th retailer.

\subsection{Model description}

It is assumed that retailers sell the item under the EOQ model with imprecise demands, therefore demands and order quantities also be fuzzy in nature for both retailers and the wholesaler.

\subsubsection{The wholesaler problem}

Wholesaler purchases $\widetilde{Q}_{w}=\left(\bar{Q}_{w}, Q_{L w}, Q_{R w}\right)$ units of item (which is fuzzy in nature) from outside suppliers and sells these units to different retailers. It is assumed that, $\delta$ fraction of the total inventory are either used for push-sale or occurs as shortage quantity. Then the following two different cases arise and corresponding wholesaler's profits are given as:

Case 1: $\delta \geq 0$, (Stock and push-sale). In this case, $\delta \widetilde{Q}$ amount of item are used for push-sale with a reduced price $S_{a}$. The inventory situation is presented in Figure 3. Therefore, wholesaler's profit $\left(\widetilde{P F}_{w}\right)$ is given by

$$
(\widetilde{P F})_{w}=S\left(\widetilde{Q}_{w}-\delta \widetilde{Q}_{w}\right)-P \widetilde{Q}_{w}+\left(S_{a}-h_{w}\right) \delta \widetilde{Q}_{w} .
$$



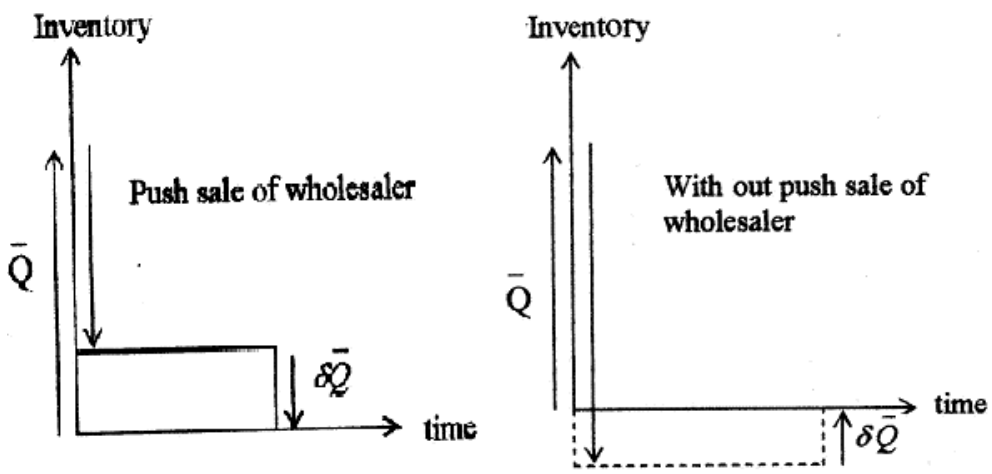

Figure 3. Wholesaler's inventory/shortage level.

Case 2: $\delta<0$, (Shortages allowed). In this case, shortages are allowed, but shortage quantities are not backlogged. This situation is also described in Figure 3. Therefore in the case of shortage the imprecise profit is:

$$
(\widetilde{P F})_{w}=S \widetilde{Q}_{w}-P \widetilde{Q}_{w}+c_{2 w}\left(\delta \widetilde{Q}_{w}\right)
$$

Hence for the both cases, the Total Profit of the wholesaler is equal to the sum of revenues from normal sale and push-sale (in Case 1) minus the purchasing and holding costs (in Case 1) and minus the shortage cost (in Case 2), i.e.,

$$
\begin{aligned}
(\widetilde{P F})_{w}= & S\left\{\widetilde{Q}_{w}-\max (\delta, 0) \widetilde{Q}_{w}\right\}-P \widetilde{Q}_{w}+\left(S_{a}-h_{w}\right)\left\{\max (\delta, 0) \widetilde{Q}_{w}\right\} \\
& +c_{2 w}\left\{\min (\delta, 0) \widetilde{Q}_{w}\right\}=\left(\overline{P F}_{w}, P F_{L w}, P F_{R w}\right),
\end{aligned}
$$

where $\overline{P F}_{w}$ is the mean profit, $\left(\overline{P F}_{w}-P F_{L w}\right)$ and $\left(P F_{R w}-\overline{P F}_{w}\right)$ are the left and right spreads about $\overline{P F}_{w}$ respectively. The centroid of the profit $\left(P F_{c}\right)_{w}$ for wholesaler is given by:

$$
\left(P F_{c}\right)_{w}=\frac{1}{3}\left(\overline{P F}_{w}+P F_{L w}+P F_{R w}\right) .
$$

\subsubsection{Retailers Problem}

Let $i$-th retailer purchases $\widetilde{Q}_{i}$ units from wholesaler at a time and start the business. Therefore in retailer's level, the fuzzy differential equation governing the stock status $\widetilde{q}_{i}(t)$ at any time $t$ is given by

$$
\frac{d \widetilde{q}_{i}}{d t}=-\widetilde{D}_{i}(t)-\theta \widetilde{q}_{i}, \quad 0 \leq t \leq t_{i} \quad \text { with boundary condition } \widetilde{q}_{i}\left(t_{i}\right)=\widetilde{c}_{i} .
$$

The fuzzy demand function is given in the form $\widetilde{D_{i}}(t)=\left(\bar{D}_{i}(t), D_{L i}(t), D_{R i}(t)\right)$, where $\bar{D}_{i}(t)=\bar{D}_{0 i} e^{-t}$ is the mean demand function at any time $t$, and $D_{L i}(t)$, $D_{R i}(t)$ are possible minimum and maximum demands with respective spreads $\Delta_{L}, \Delta_{R}$ to the constant $\bar{D}_{0 i}$. To describe the model, the mean demand is assumed to vary exponentially with time $t$, i.e.

$$
\bar{D}_{i}(t)=\bar{D}_{0 i} e^{-t}, \quad D_{L i}(t)=\left(\bar{D}_{0 i}-\Delta_{L}\right) e^{-t}, \quad D_{R i}(t)=\left(\bar{D}_{0 i}+\Delta_{R}\right) e^{-t} \text {. }
$$


Now the different sub-cases inventory situation depending on the values of $\widetilde{c_{i}}$ are given by

Sub-case 1. The retailer has no inventory at time $t_{i}$. Pictorial representation of this inventory situation is presented in Fig. 4.

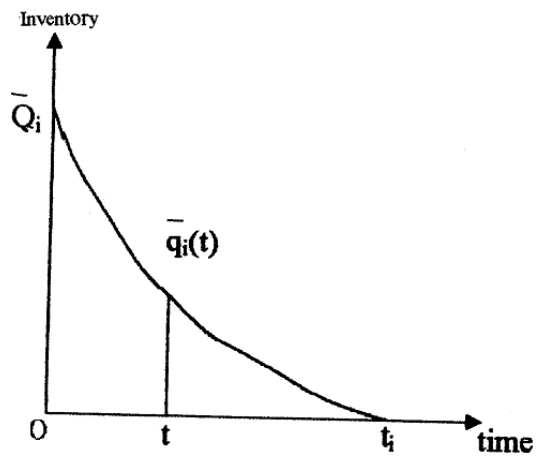

Figure 4. Inventory ends with neither shortage nor stock.

Sub-case 2. When some excess inventory are in stock at time $t_{i}$, they are sold immediately at a reduced price $s_{a i}$. This inventory pattern is presented in Fig. 5.

Sub-case 3. When some shortages occur at time $t_{i}$, there is a penalty cost. This inventory pattern is presented in Fig. 6.

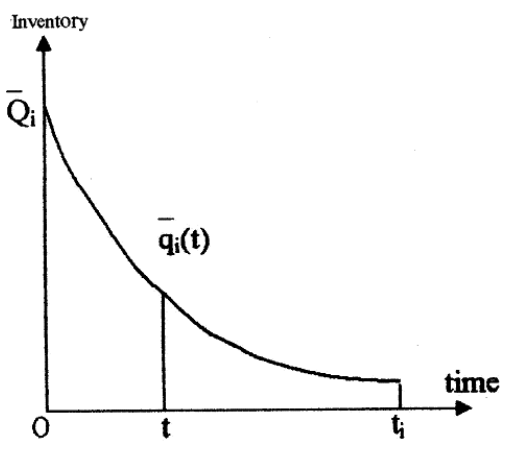

Figure 5. Inventory ends with stock.

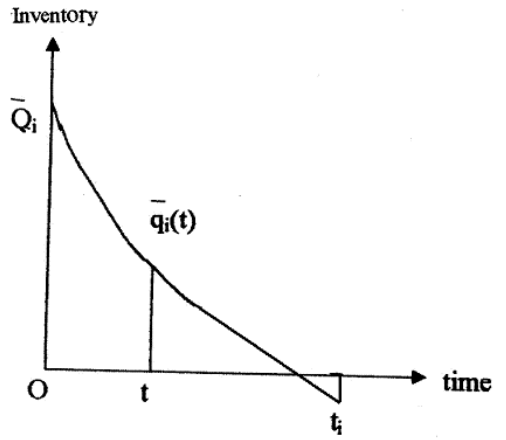

Figure 6. Inventory ends with shortage

For finding a solution we use Procedure 2. Let us consider the demand function $\bar{D}_{i}(t)=\bar{D}_{0 i} e^{-t}$, its membership value is 1 . Then the reduced crisp differential equation

$$
\frac{d \bar{q}_{i}}{d t}=-\bar{D}_{i}(t)-\theta \bar{q}_{i} \quad 0 \leq t \leq t_{i} \quad \text { with boundary condition, } \bar{q}_{i}\left(t_{i}\right)=\bar{c}_{i}
$$


has a solution

$$
\begin{aligned}
\bar{q}_{i}(t)=\bar{c}_{i} e^{\theta\left(t_{i}-t\right)} & +\left(\bar{D}_{0 i} \frac{e^{-\theta t}}{1-\theta}\right)\left(e^{-t(1-\theta)}-e^{-t_{i}(1-\theta)}\right) \\
& +\left(\bar{D}_{0 i} \frac{e^{-t}}{1-\theta}\right)\left(1-e^{-(1-\theta)\left(t_{i}-t\right)}\right)
\end{aligned}
$$

Then we fuzzify $\bar{q}_{i}$ with the assumptions $\widetilde{c}_{i} \geq 0, \widetilde{D}_{o i}>0$ since

$$
\frac{d \bar{q}_{i}}{d \bar{c}_{i}}=e^{\theta\left(t_{i}-t\right)}>0, \quad \frac{d \bar{q}_{i}}{d \bar{D}_{o i}}=\frac{1-e^{(1-\theta) t_{i}}}{1-\theta}>0
$$

and after fuzzifying $\bar{q}_{i}$, we get the fuzzy inventory level at any time $t$ as:

$$
\begin{aligned}
\widetilde{q}_{i}(t) & =\left(\bar{q}_{i}(t), q_{L i}(t), q_{R i}(t)\right), \quad 0 \leq t \leq t_{i} \\
q_{L i}(t) & =\min \left\{\bar{q}_{i}\left(t, c_{i}, D_{0 i}\right): c_{i} \in \widetilde{c}_{i}[\alpha], D_{0 i} \in \widetilde{D}_{0 i}[\alpha]\right\}, \\
q_{R i}(t) & =\max \left\{\bar{q}_{i}\left(t, c_{i}, D_{0 i}\right): c_{i} \in \widetilde{c}_{i}[\alpha], D_{0 i} \in \widetilde{D}_{0 i}[\alpha]\right\} .
\end{aligned}
$$

Particularly, when $\alpha=0$ we get the solution following Procedure 1 .

Inventory cost and Components. At the beginning of the interval $\left(0, t_{i}\right)$ fuzzy order quantity to each retailer is $\widetilde{Q_{i}}=\left(\bar{Q}_{i}, Q_{L i}, Q_{R i}\right), 0 \leq t \leq t_{i}$, where

$$
\begin{aligned}
& \bar{Q}_{i}=\bar{c}_{i} e^{\theta t_{i}}+\frac{\bar{D}_{0 i}}{1-\theta}\left(1-e^{-t_{i}(1-\theta)}\right), \\
& Q_{L i}=\min \left\{\bar{Q}_{i}\left(c_{i}, D_{0 i}\right): c_{i} \in \widetilde{c}_{i}[\alpha], D_{0 i} \in \widetilde{D}_{0 i}[\alpha]\right\}, \\
& Q_{R i}=\max \left\{\bar{Q}_{i}\left(c_{i}, D_{0 i}\right): c_{i} \in \widetilde{c}_{i}[\alpha], D_{0 i} \in \widetilde{D}_{0 i}[\alpha]\right\} .
\end{aligned}
$$

Therefore the total deterioration $\widetilde{G}_{i}$ during the period is:

$$
\begin{aligned}
\widetilde{G_{i}}=\widetilde{Q_{i}}-\int_{0}^{t_{i}} \widetilde{D}_{i}(t) d t=\widetilde{Q_{i}}+\left(\bar{D}_{0 i} e^{-t_{i}}\left(1-e^{t_{i}}\right),\right. \\
\left.\left(D_{0 i}-\Delta_{L}\right) e^{-t_{i}}\left(1-e^{t_{i}}\right),\left(D_{0 i}+\Delta_{R}\right) e^{-t_{i}}\left(1-e^{t_{i}}\right)\right)
\end{aligned}
$$

and the inventory holding cost, $\widetilde{H}_{i}$ during the period $\left[0, t_{i}\right]$ is:

$$
\widetilde{H_{i}}=\int_{0}^{t_{i}} h_{i} \widetilde{q}_{i}(t) d t=\left(\bar{H}_{i}, H_{L i}, H_{R i}\right),
$$

where

$$
\begin{aligned}
& \bar{H}_{i}=\bar{c}_{i} \frac{h_{i}}{\theta}\left(e^{\theta t_{i}}-1\right)+\frac{h_{i} \bar{D}_{0 i}}{1-\theta}\left\{\left(1-e^{-t_{i}}\right)+\frac{1}{\theta} e^{-t_{i}}\left(1-e^{\theta t_{i}}\right)\right\}, \\
& H_{L i}(t)=\min \left\{\bar{H}_{i}\left(c_{i}, D_{0 i}\right): c_{i} \in \widetilde{c}_{i}[\alpha], D_{0 i} \in \widetilde{D}_{0 i}[\alpha]\right\}, \\
& H_{R i}(t)=\max \left\{\bar{H}_{i}\left(c_{i}, D_{0 i}\right): c_{i} \in \widetilde{c}_{i}[\alpha], D_{0 i} \in \widetilde{D}_{0 i}[\alpha]\right\} .
\end{aligned}
$$


Also the sale proceeds $\widetilde{S P}_{i}$ of the $i$-th retailer is (see Appendix A):

$$
\widetilde{S P_{i}}=\int_{0}^{t_{i}} s_{i}(t) \widetilde{D_{i}}(t) d t=\left(\overline{S P}_{i}, S P_{L i}, S P_{R i}\right)
$$

Therefore the total profit $\widetilde{P F}_{i}$ of the inventory system of $i$-th retailer is:

$$
\begin{aligned}
\left(\widetilde{P F_{i}}\right) & =\left\{\widetilde{S P_{i}}-S \min \left\{\widetilde{Q}_{i},\left(\widetilde{Q}_{i}-\eta_{i} \delta \widetilde{Q}_{i}\right)\right\}-S_{a} \max \left\{0, \eta_{i} \delta \widetilde{Q}_{i}\right\}-\widetilde{H}_{i}-g_{i} \widetilde{G}_{i}\right\} \\
& =\left(\overline{P F}_{i}, P F_{L i}, P F_{R i}\right),
\end{aligned}
$$

where

$$
\begin{gathered}
\overline{P F}_{i}=\left\{\overline{S P}_{i}-S \min \left\{\bar{Q}_{i},\left(\bar{Q}_{i}-\eta_{i} \delta \bar{Q}_{i}\right)\right\}-S_{a} \max \left\{0, \eta_{i} \delta \bar{Q}_{i}\right\}-\bar{H}_{i}-g_{i} \bar{G}_{i}\right\}, \\
P F_{L i}=\left\{S P_{L i}-S \min \left\{Q_{R i},\left(Q_{R i}-\eta_{i} \delta Q_{R i}\right)\right\}-S_{a} \max \left\{0, \eta_{i} \delta Q_{R i}\right\}\right. \\
-H_{R i}-g_{i} G_{R i}, \\
P F_{R i}=\left\{S P_{R i}-S \min \left\{Q_{L i},\left(Q_{L i}-\eta_{i} \delta Q_{L i}\right)\right\}-S_{a} \max \left\{0, \eta_{i} \delta \cdot Q_{L i}\right\}\right. \\
-H_{L i}-g_{i} G_{L i} .
\end{gathered}
$$

Therefore the centroid of the profit to each retailer is equal to

$$
\left(P F_{c}\right)_{i}=\frac{1}{3}\left(\overline{P F}_{i}+P F_{L i}+P F_{R i}\right)
$$

Now the problem is to find out the quantities to be delivered/distributed to each retailers, from the wholesaler which maximizes the profits of wholesaler and retailers respectively.

$$
\begin{array}{rccc}
\text { i.e., maximize } & \left(P F_{c}\right)_{w} & \text { (from equation (3.1)) } & \text { and } \\
\text { maximize } & \left(P F_{c}\right)_{i} & \text { (from equation (3.9)) } & \forall i
\end{array}
$$

Here as wholesaler and retailers are different persons, their interests i.e., profits are maximized separately. The problem posed by $(3.10)-(3.11)$ is a multiobjective problem. Normally, a multi-objective problem is first converted to an equivalent single objective problem using e.g. the Utility Function Method (UFM), Goal programming method or Goal Attainment method. Weight sum method is a particular case of UFM. There is a soft computing method, MOGA developed by Deb et al. [5], which is applied to solve multi-objective problem directly. It optimizes the objectives simultaneously giving equal importance to each objective and gives a set of Pareto optimum solutions. However, depending on the situation, if DM prefers to give different importance to different objectives, then IFDM technique is most suitable.

\section{Multi-objective Genetic Algorithm}

\subsection{Non-dominated sorting of a population}

Most evolutionary multi-objective optimization algorithms require to find only the best non-dominated front in a population. These algorithm classify the population into two sets. i.e. the non-dominated set and the remaining dominated 
set. However, there exist some algorithms which require the entire population to be classified into various non-dominated levels. In such algorithms, the population needs to be sorted according to an ascending level of non-domination. The best non-dominated solutions are called non-dominated solutions of level 1. In order to find solutions for the next level of non-domination, there is a simple procedure which is usually followed. Once the best non-dominated set is identified, they are temporarily disregarded from the population. The nondominated solutions of the remaining populations are then found and are called non-dominated solutions of level 2. In order to find the non-dominated solutions of level 3, all non-dominated solutions of levels 1 and 2 are disregarded and new non-dominated solutions are found. This procedure is continued until all population members are classified into a non-dominated level. We note, that non-dominated solutions of level 1 are better than non-dominated solutions of level 2, and so on. The following approach describes a step-by-step procedure for non-dominated sorting.

- Step 1: Set all non-dominated sets $P_{j},(j=1,2, \ldots)$ as empty sets. Set non-domination level counter $j=1$.

- Step 2: Use any one of the Approaches 1 to 3 to find the non-dominated set $P^{\prime}$ of population $P$.

- Step 3: Update $P_{j}=P^{\prime}$ and $P=P \backslash P^{\prime}$.

- Step 4: If $P \neq \emptyset$, increment $j$ by one and go to Step 2. Otherwise, stop and declare all non-dominated sets $P_{i}$, for $i=1,2, \ldots, j$.

\subsubsection{Fast nondominated sorting approach(FNDS)}

In this case, for each solution we calculate two entities: (1) domination count $n_{p}$, the number of solutions which dominate the solution $X_{p}$, and (2) $S_{p}$, a set of solutions that the solution $X_{p}$ dominates. All solutions in the first nondominated front will have their domination count as zero. Now, for each solution $X_{p}$ with $n_{p}=0$, we visit each member $X_{q}$ of its set $S_{p}$ and reduce its domination count by one. In doing so, if for any member $X_{q}$ the domination count becomes zero, we put it in a separate list $Q$. These members belong to the second nondominated front. Now, the above procedure is continued with each member of $Q$ and the third front is identified. This process continues until all fronts are identified. For each solution $X_{p}$ in the second or higher level of nondomination, the domination count $n_{p}$ can be at most POPSIZE - 1 . Thus, each solution $X_{p}$ will be visited at most POPSIZE - 1 times before its domination count becomes zero. At this point, the solution is assigned a nondomination level and will never be visited again. The algorithm of FNDS is given below:

\section{Procedure FNDS $(\mathrm{P})$}

begin

for each $X_{p} \in P$ 


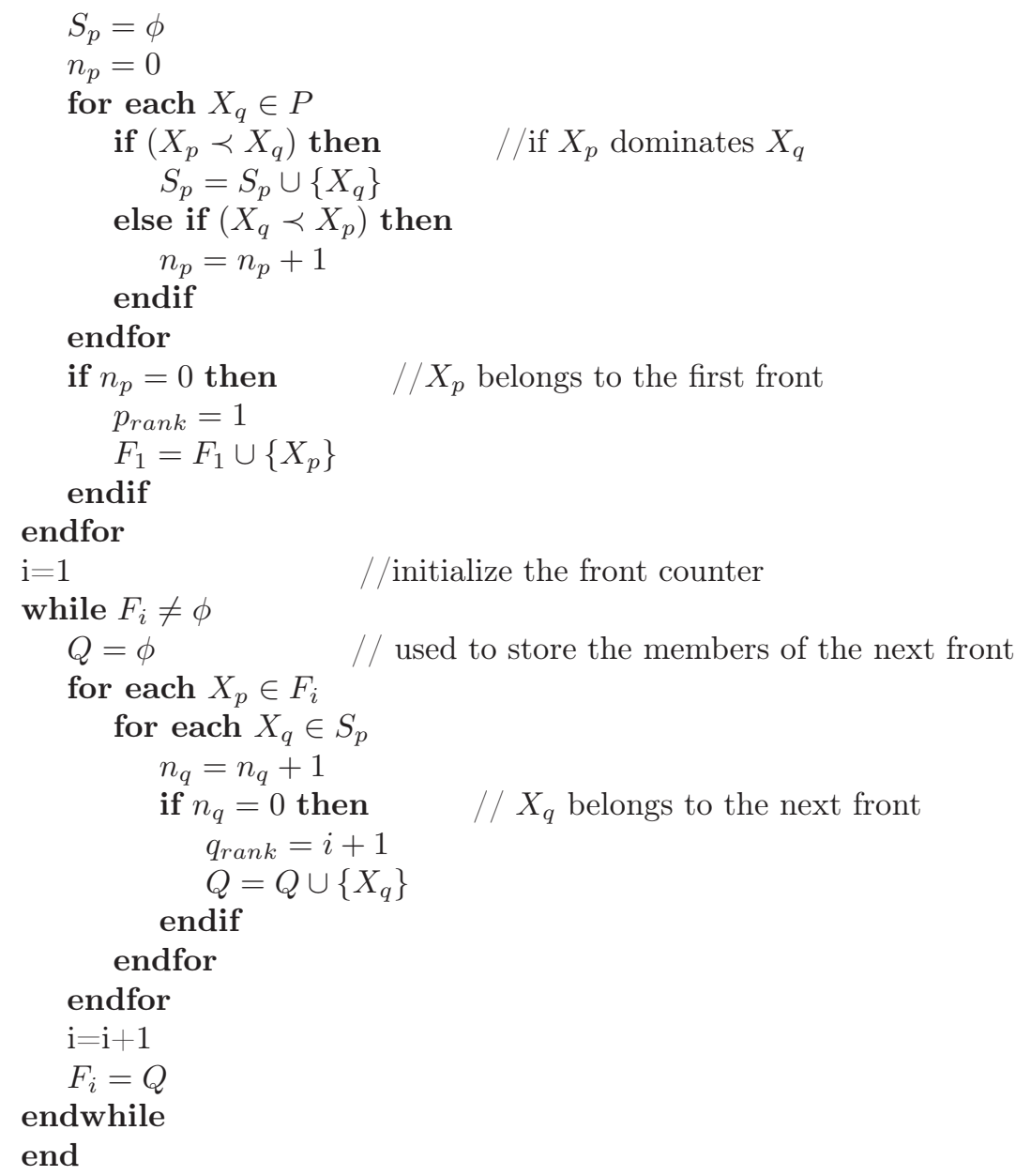

\section{Procedure MOGA}

begin

$t \leftarrow 0$

initialize Population $\mathrm{P}(\mathrm{t})$

evaluate Population $\mathrm{P}(\mathrm{t})$

while(not terminate-condition) do

begin

$t \leftarrow t+1$

select parents from Population $\mathrm{P}(\mathrm{t}-1)$

create offsprings $\mathrm{C}(\mathrm{t})$ by altering(using crossover and mutation) the selected parents

$P_{1}(t) \leftarrow P(t-1) \cup C(t)$

split $P_{1}(t)$ into nondominated fronts $F_{1}, F_{2}, \ldots$ using FNDS

$P_{2}(t) \leftarrow F_{1} \cup F_{2} \cup \ldots \cup F_{i}$, where $i$ is the greatest integer such that order of $P_{2}(t) \leq P O P S I Z E$

if order of $P_{2}(t)<P O P S I Z E$ then 
select (POPSIZE - order of $\left.P_{2}(t)\right)$ number of chromosomes from the front $F_{i+1}$ and then add these to $P_{2}(t)$

$$
P(t) \leftarrow P_{2}(t)
$$

evaluate Population $\mathrm{P}(\mathrm{t})$

$$
\text { end }
$$

end

\section{$5 \quad$ Numerical Examples}

To illustrate the proposed inventory model, a system of one wholesaler and two retailers is considered where wholesaler sells the item to retailers instantaneously after replenishment and retailers again sells those to the customers as a single period inventory model. The following input data are considered for illustration:

$$
\begin{aligned}
& \widetilde{c}_{1}=(0,-0.2,0.3), \widetilde{c}_{2}=(0,-0.3,0.2), \quad \alpha=0.2, \quad P=\$ 22, \quad S=\$ 40, \\
& S_{a}=\$ 35, \quad s_{01}=\$ 50, \quad s_{02}=\$ 52, \quad D_{01}=130 \text { units, } D_{02}=135 \text { units, } \\
& b_{1}=0.05, \quad b_{2}=0.05, \quad \theta=0.08, \quad \Delta_{L}=0.05, \quad \Delta_{R}=0.07, \\
& h_{1}=\$ 2.40, \quad h_{2}=\$ 2.50, \quad h_{w}=\$ 1.0, \quad c_{2 w}=\$ 0.4 .
\end{aligned}
$$

\subsection{Solution of Fuzzy Inventory Model without DM's preference by MOGA:}

The problem represented by (3.10) and (3.11) is solved by MOGA and the results are presented in Tables 1 and 2 . Wholesaler's order quantity is equal to a sum of both retailers order quantities.

Table 1. Optimum order quantities and profits obtained by Proc 2 via MOGA.

\begin{tabular}{cccccc}
\hline cases & $\begin{array}{c}\text { Ret-1's } \\
\text { Ord.Quan. }\end{array}$ & $\begin{array}{c}\text { Ret-2's } \\
\text { Ord.Quan. }\end{array}$ & $\begin{array}{c}\text { Whol's } \\
\text { Profit }(\$)\end{array}$ & $\begin{array}{c}\text { Ret-1's } \\
\text { Profit }(\$)\end{array}$ & $\begin{array}{c}\text { Ret-2's } \\
\text { Profit }(\$)\end{array}$ \\
\hline \multirow{3}{*}{$\delta=0$} & $34.02 ; 35.69 ; 37.98$ & $37.90 ; 39.29 ; 41.67$ & 1575.50 & 3756.61 & 3998.48 \\
& $33.40 ; 35.68 ; 37.10$ & $40.04 ; 41.65 ; 44.22$ & 1574.39 & 3764.92 & 4121.83 \\
& $34.20 ; 36.68 ; 38.74$ & $37.58 ; 40.83 ; 42.23$ & 1538.17 & 3773.62 & 4110.64 \\
\hline \multirow{3}{*}{$\delta=5 \%$} & $33.14 ; 35.63 ; 37.18$ & $36.95 ; 37.98 ; 40.05$ & 1390.54 & 3819.38 & 4438.58 \\
& $34.87 ; 36.00 ; 38.13$ & $35.53 ; 37.88 ; 39.80$ & 1388.71 & 3803.57 & 4458.65 \\
& $33.2 ; 35.87 ; 37.35$ & $36.45 ; 38.20 ; 39.76$ & 1387.43 & 3777.99 & 4454.54 \\
\hline
\end{tabular}

\subsection{Solution of Fuzzy Inventory Model without DM's preference by IFDM}

The problem given in (3.10) and (3.11) is now solved by IFDM method and the results are presented in the following pay-off matrix of the profit functions 
Table 2. Optimum order quantities and profits obtained by Proc 1 via MOGA.

\begin{tabular}{cccccc}
\hline cases & $\begin{array}{c}\text { Ret-1's } \\
\text { Ord.Quan. }\end{array}$ & $\begin{array}{c}\text { Ret-2's } \\
\text { Ord.Quan. }\end{array}$ & $\begin{array}{c}\text { Whol's } \\
\text { Profit (\$) }\end{array}$ & $\begin{array}{c}\text { Ret-1's } \\
\text { Profit }(\$)\end{array}$ & $\begin{array}{c}\text { Ret-2's } \\
\text { Profit (\$) }\end{array}$ \\
\hline \multirow{3}{*}{$\delta=0$} & $33.45 ; 35.69 ; 39.79$ & $37.12 ; 39.29 ; 42.16$ & 1571.55 & 3750.12 & 3991.14 \\
& $32.22 ; 35.68 ; 37.76$ & $39.24 ; 41.65 ; 44.75$ & 1569.03 & 3760.42 & 4117.88 \\
& $33.40 ; 36.68 ; 39.80$ & $36.24 ; 40.83 ; 44.28$ & 1533.84 & 3772.36 & 4109.34 \\
\hline \multirow{3}{*}{$\delta=5 \%$} & $32.85 ; 35.63 ; 38.71$ & $36.15 ; 37.98 ; 42.28$ & 1390.00 & 3817.35 & 4435.20 \\
& $34.02 ; 36.00 ; 38.76$ & $35.00 ; 37.88 ; 40.35$ & 1385.00 & 3801.24 & 4455.24 \\
& $33.02 ; 35.87 ; 38.73$ & $36.10 ; 38.20 ; 40.97$ & 1383.57 & 3773.25 & 4451.45 \\
\hline
\end{tabular}

$P F_{k}$ for $\delta=0$ and $\delta=5$ :

$$
\left(\begin{array}{ccc}
P F_{c w} & P F_{c 1} & P F_{c 2} \\
2401.19 & 2258.54 & 2732.891 \\
1062.28 & 10604.70 & 2924.00 \\
1125.08 & 2446.10 & 10412.38
\end{array}\right),\left(\begin{array}{ccc}
P F_{c w} & P F_{c 1} & P F_{c 2} \\
2372.20 & 2258.54 & 2760.73 \\
921.52 & 10867.66 & 4181.63 \\
993.55 & 2558.55 & 10632.34
\end{array}\right) .
$$

In addition to the above input data, we consider lower and upper bounds for the profit function which are within the lower and upper bounds of the pay-off matrix. This information is presented in Table 3.

Table 3. Input data for lower and upper values of $P F_{k}$ 's.

\begin{tabular}{ccccc}
\hline Member & \multicolumn{2}{c}{$\delta=0$} & \multicolumn{2}{c}{$\delta=5 \%$} \\
& Lower Upper & \multicolumn{2}{c}{ Lower Upper } \\
\hline$P F_{c w}$ & 1090.00 & 2020.00 & 1170.00 & 2040.00 \\
$P F_{c 1}$ & 2960.00 & 4560.00 & 2760.00 & 5260.00 \\
$P F_{c 2}$ & 2920.00 & 4010.00 & 2830.30 & 5100.00 \\
\hline
\end{tabular}

With the above values, the membership functions of the objective functions were formed of the types as given in Table 4 .

Table 4. Possible types of MF for objective functions.

\begin{tabular}{cl}
\hline Objective functions & Type of membership functions \\
\hline$P F_{c w}$ & Type-I or Type-II or Type-III \\
$P F_{c 1}$ & Type-I or Type-II or Type-III \\
$P F_{c 2}$ & Type-I or Type-II or Type-III \\
\hline
\end{tabular}

At the beginning, analysis is performed to find optimum values with the membership functions for $P F_{c w}, P F_{c 2}$ as linear (Type I) and $P F_{c 1}$ as Quadratic (Type II). The optimum values of $\alpha$ are $\alpha^{*}=0.643$ (for $\delta=0$ ) and $\alpha^{*}=0.779$ (for $\delta=5 \%$ ). With these values of $\alpha^{*}$, the objective functions are optimized to their corresponding optimum order quantities and profits. The Generalised 
Reduced Gradient method was used for optimization. The obtained results are presented in Table 5.

Table 5. Optimal results with priority (importance) to different objective functions.

\begin{tabular}{ccccccc}
\hline cases & $\begin{array}{c}\text { Import. } \\
\text { Mem. }\end{array}$ & $\begin{array}{c}\text { Ret-1's } \\
\text { Ord.Quan. }\end{array}$ & $\begin{array}{c}\text { Ret-2's } \\
\text { Ord.Quan. }\end{array}$ & $\begin{array}{c}\text { Whol's } \\
\text { Profit }(\$)\end{array}$ & $\begin{array}{c}\text { Ret-1's } \\
\text { Profit }(\$)\end{array}$ & $\begin{array}{c}\text { Ret-2's } \\
\text { Profit }(\$)\end{array}$ \\
\hline \multirow{3}{*}{$\delta=0$} & $P F_{c w}$ & 39.69 & 38.76 & $\mathbf{1 6 4 0 . 9 4}$ & 3561.87 & 3468.82 \\
& $P F_{c 1}$ & 38.73 & 37.23 & 1508.12 & $\mathbf{4 0 0 9 . 6 8}$ & 3561.87 \\
& $P F_{c 2}$ & 38.76 & 37.21 & 1507.45 & 3468.82 & $\mathbf{4 1 1 9 . 0 0}$ \\
\hline \multirow{4}{*}{$\delta=5 \%$} & $P F_{c w}$ & 39.69 & 38.76 & $\mathbf{1 4 6 6 . 7 6}$ & 3612.25 & 3839.56 \\
& $P F_{c 1}$ & 38.73 & 37.23 & 1355.60 & $\mathbf{4 1 5 0 . 4 0}$ & 3839.05 \\
& $P F_{c 2}$ & 38.76 & 37.21 & 1354.91 & 3611.41 & $\mathbf{4 2 4 0 . 6 4}$ \\
\hline
\end{tabular}

\section{Discussion}

Table 1 gives the optimum order quantities and profits for the wholesaler and retailers with equal priority to each of them. Moreover, through MOGA, a set of near optimum solutions for wholesaler and retailers is presented. Here DM may opt the optimum or any one of the near optimum solutions according to his/her convenience considering the real-life situation. In (5.1), pay-off matrix i.e., lower and upper boundaries of profit function for the system are presented. It is noted that corresponding optimum values in Table 1 stay within these extreme values. Values in Table 3 are used to construct the membership functions for the profit objectives. Here again DM plays a crucial role. Depending upon his/her experience on the various objective functions, he/she selects the type of the membership functions.

In Table 5, optimum values have been displayed for particular type of membership functions of the objectives. Comparing the results in Table 1 (with equal importance) and Table 5(with varied importance), it is observed that the profits for wholesaler do not differ much. For the case with $\delta=0$, maximum profits for wholesaler are $\$ 1575.5$ and $\$ 1640.94$, respectively. Similar, in the case of retailer- 2 those values are $\$ 4119.00$ and $\$ 4263.37$. But, in the case of retailer-1, maximum values differ considerably as the maximum profits are $\$ 3773.62$ in MOGA and $\$ 4009.68$ in IFDM. This is because we considered the quadratic membership function for retailer-1.

The same features are observed for the case with $\delta=5 \%$. It also reveals that, when $5 \%$ push-sale is considered, the wholesaler loses nearly $11 \%$ of his profit. In this situation each retailer's profit increases between $4 \%$ to $5 \%$ for the given input data.

\section{Conclusion}

In this paper, a realistic bi-level inventory problem with imprecise demand and time dependent reducing selling price has been presented. Here, the imprecise 
demands of the customers at different locations vary with time and are met through the retailer at that location. So the governing differential equations and the corresponding stock status of the retailers are fuzzy in nature which are solved in fuzzy sense. After that, the profit functions of wholesaler and different retailers are formulated and these are also fuzzy in nature. The fuzzy objective functions are represented in terms of mean function, left and right spread functions and then defuzzified by the centroid of corresponding objective function.

The problem is solved using both MOGA and IFDM technique. The IFDM technique helps the DM to get the solution which suits him/her best. For the first time, a real-life, inventory control problem has been formulated at bilevel with time-dependent imprecise demand. The solution technique is based on a fuzzy differential equation, which is formulated for the inventory control system.

Finally, future research in this direction should consider the extension of the present approach to the inventory control system with finite time, quantity discount, imprecise inventory cost.

\section{Appendix-A}

It is define that,

$$
\begin{aligned}
\widetilde{S P_{j}}=\left(\overline{S P}, S P_{L}, S P_{R}\right)_{j} & =\int_{0}^{t_{j}} s_{j}(t) \widetilde{D_{j}}(t) d t+s_{a j} \widetilde{c}_{j} \\
& =\int_{0}^{t_{j}} s_{0 j}\left(1-\frac{b j}{100}[t]\right) \widetilde{D_{j}}(t) d t+s_{a j} \widetilde{c}_{j} .
\end{aligned}
$$

Therefore, for $i$ equal to the integer part of $t_{j}$

$$
\begin{aligned}
\overline{S P} & =\int_{0}^{t_{j}} s_{0 j}\left(1-\frac{b_{j}}{100}[t]\right) \bar{D}_{0 j} e^{-t} d t+s_{a j} \widetilde{c}_{j} \\
= & s_{0 j} \bar{D}_{0 j}\left\{\sum_{k=1}^{i} \int_{k-1}^{k}\left(1-\frac{b_{j}}{100}(k-1) e^{-t} d t+\int_{i}^{t_{j}}\left(1-\frac{b_{j}}{100} i\right) e^{-t} d t\right\}+s_{a j} \widetilde{c}_{j}\right. \\
= & s_{0 j} \bar{D}_{0 j}\left\{\sum_{k=1}^{i}\left(1-\frac{b_{j}}{100}(k-1) \int_{k-1}^{k} e^{-t} d t+\left(1-\frac{b_{j}}{100} i\right) \int_{i}^{t_{j}} e^{-t} d t\right\}+s_{a j} \widetilde{c}_{j}\right. \\
= & s_{0 j} \bar{D}_{0 j}\left\{\left(1-\frac{b_{j}}{100} i\right)\left(e^{-i}-e^{-t_{j}}\right)+\sum_{k=1}^{i}\left(1-\frac{b_{j}}{100}(k-1) e^{-k}(e-1)\right\}+s_{a j} \widetilde{c}_{j},\right. \\
S P_{L} & =\int_{0}^{t_{j}} s_{0 j}\left(1-\frac{b_{j}}{100}[t]\right)\left(\bar{D}_{0 j}-\Delta_{L}\right) e^{-t} d t=\overline{S P}-\Delta_{L} s_{0 j} \\
& \times \int_{0}^{t_{j}}\left(1-\frac{b_{j}}{100}[t]\right) e^{-t} d t=\overline{S P}-\Delta_{L} s_{0 j} \\
& \times\left(\left(1-e^{-t_{j}}\right)-\frac{b_{j}}{100}\left\{\sum_{a=1}^{\left[t_{j}\right]}(a-1) e^{a}(e-1)+\left[t_{j}\right]\left(e^{-\left[t_{j}\right]}-e^{-t_{j}}\right)\right\}\right)
\end{aligned}
$$


and

$$
\begin{aligned}
S P_{R} & =\int_{0}^{t_{j}} s_{0 j}\left(1-\frac{b_{j}}{100}[t]\right)\left(\bar{D}_{0 j}+\Delta_{R}\right) e^{-t} d t=\overline{S P}+\Delta_{R} s_{0 j} \\
& \times \int_{0}^{t_{j}}\left(1-\frac{b_{j}}{100}[t]\right) e^{-t} d t=\overline{S P}+\Delta_{R} s_{0 j}\left(\left(1-e^{-t_{j}}\right)\right. \\
& \left.-\frac{b_{j}}{100}\left\{\sum_{a=1}^{\left[t_{j}\right]}(a-1) e^{a}(e-1)+\left[t_{j}\right]\left(e^{-\left[t_{j}\right]}-e^{-t_{j}}\right)\right\}\right) .
\end{aligned}
$$

\section{References}

[1] R.E. Bellman and L.A. Zadeh. Decision making in a fuzzy environment. Management Science, 17(4):B141-B164, 1970.

[2] J. J. Buckley. Theory Feuring. Fuzzy differential equations. Fuzzy Sets and Systems, 110:43-54, 2000.

[3] H.J. Chang and C.Y. Dye. An EOQ model for deteriorating items with timevarying demand and partial backlogging. Journal of Operational Research, 50:1176-1182, 1999.

[4] J. Chiang, J.S. Yao and H.M. Lee. Fuzzy inventory with backorder defuzzification by signed distance method. Journal of Information Science and Engineering, 21:671-694, 2005.

[5] K. Deb, A. Pratap, S. Agarwal and T. Meyarivan. A fast and elitist multiobjective genetic algorithm. NSGA-II, IEEE Transactionary Computation, 6(2), 2002.

[6] J. Dey, S. Kar and M. Maiti. An interactive method for inventory control with fuzzy lead time and dynamic demand. European Journal of Operation Research, 167:381-397, 2005.

[7] W.A. Donaldson. Inventory replenishment policy for a linear trend in demand-an analytical solution. Operational Research Quarterly, 28:663-670, 1977.

[8] D. Dubois and H. Prade. Fuzzy sets and system-Theory and application. Academic Publishers. New York, 1980.

[9] M. Friedman, M. Ming and A. Kandel. On the validity of Peano theorem for Fuzzy differential equations. Fuzzy Sets and Systems 86, pp. 331-334, 1997.

[10] G. Gallego and I. Moon. The distribution free newsboy problem: review and extensions. Journal of the Operational Research Society, 44(8):825-834, 1993.

[11] F. Harris. Operations and Cost-Factory management series. A.W. Shaw Co. Chicago,IL, 1915.

[12] H.M. Lee and J. S. Yao. Economic order quantity in fuzzy sense for inventory without backorder model. Fuzzy Sets and Systems, 105:13-31, 1999.

[13] S. Mondal and M. Maiti. Multi-item fuzzy eoq models using genetic algorithm. Computers and Industrial Engineering, 44:105-117, 2002.

[14] M. Sakawa and H.Yano. An interactive fuzzy decision making for multi-objective non-linear programming using augmented minimax problems. Fuzzy Sets and Systems, 20:31-41, 1986. 
[15] S. Seikkala. On the fuzzy initial value problem. Fuzzy Sets and Systems, 24:319330, 1987.

[16] L. A. Zadeh. Fuzzy sets. Information and Control, 8:338-356, 1965.

[17] H. J. Zimmermann. Fuzzy Set Theory. Kluwer Academic Publishers. Boston/ Dordrecht/ London, 1996. 